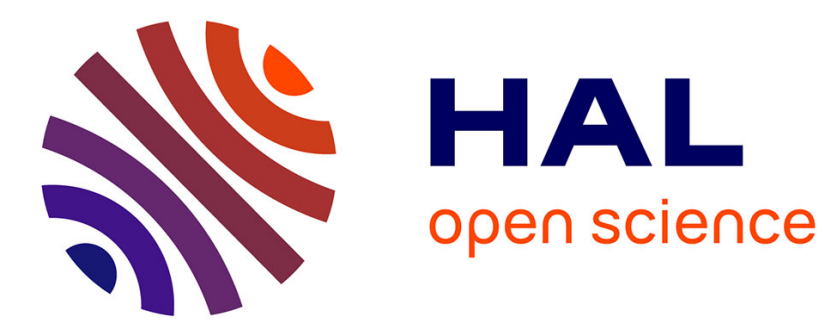

\title{
Increase in depth of field taking into account deconvolution by optimization of pupil mask
}

Frederic Diaz, François Goudail, Brigitte Loiseau, Jean-Pierre Huignard

\section{To cite this version:}

Frederic Diaz, François Goudail, Brigitte Loiseau, Jean-Pierre Huignard. Increase in depth of field taking into account deconvolution by optimization of pupil mask. Optics Letters, 2009, 34 (19), pp.2970-2972. hal-00746985

\section{HAL Id: hal-00746985 \\ https://hal-iogs.archives-ouvertes.fr/hal-00746985}

Submitted on 30 Oct 2012

HAL is a multi-disciplinary open access archive for the deposit and dissemination of scientific research documents, whether they are published or not. The documents may come from teaching and research institutions in France or abroad, or from public or private research centers.
L'archive ouverte pluridisciplinaire HAL, est destinée au dépôt et à la diffusion de documents scientifiques de niveau recherche, publiés ou non, émanant des établissements d'enseignement et de recherche français ou étrangers, des laboratoires publics ou privés. 


\title{
Increase in depth of field taking into account deconvolution by optimization of pupil mask
}

\author{
Frédéric Diaz, ${ }^{1,2, *}$ François Goudail, ${ }^{2}$ Brigitte Loiseaux, ${ }^{1}$ and Jean-Pierre Huignard ${ }^{1}$ \\ ${ }^{1}$ Thales Research \& Technology, 1 Avenue Augustin Fresnel, 91767 Palaiseau CEDEX, France \\ ${ }^{2}$ Laboratoire Charles Fabry de l'Institut d'Optique, CNRS, Université Paris-Sud, Campus Polytechnique, \\ RD 128, 91127 Palaiseau CEDEX, France \\ *Corresponding author: frederic.diaz@thalesgroup.com
}

Received June 8, 2009; revised August 12, 2009; accepted August 24, 2009;

posted August 31, 2009 (Doc. ID 112522); published September 24, 2009

\begin{abstract}
We consider optimization of hybrid imaging systems including a phase mask for enhancing the depth of field and a digital deconvolution step. We propose an image quality criterion that takes into account the variability of the system's point-spread function along the expected defocus range and the noise enhancement induced by deconvolution. Considering the classical cubic phase mask as an example, we show that the optimization of this criterion may lead to filter parameters that are significantly different from those usually proposed to ensure the strict invariance of the PSF. () 2009 Optical Society of America

OCIS codes: $110.7348,110.4280,110.4100,100.1830$.
\end{abstract}

In traditional imaging systems, the purpose of the optics is to provide an image as sharp as possible. This image might then be postprocessed to reveal information relevant to the aimed application, but optimization of the optics and of the postprocessing stage are performed separately. Conversely, in a hybrid imaging approach [1], the optics and the postprocessing step are designed together: the image provided by the camera is generally blurred and becomes sharp only after the postprocessing. Such a design allows one to relax the constraints on the imaging lenses and to obtain a better performance at lower fabrication cost. Using this principle, Cathey and Dowski proposed to include a cubic phase mask that makes the point-spread function (PSF) insensitive to defocus and an appropriate postprocessing step to recover the quality of the image [1,2]. Different types of phase masks have been proposed to enhance the depth of field, such as exponential [3], logarithmic [4], polynomial [5], and rational phase masks [6]. These masks depend on parameters that are optimized with respect to an aimed application.

In such an approach, two effects can degrade the quality of the final image. The first one is the variation in the PSF with respect to defocus, whereas the deconvolution filter is unique. The second one is the noise enhancement owing to deconvolution, which increases as the imaging system has a low spatial bandpass behavior. With usual phase masks, the more invariant to defocus the PSF is, the more they degrade the performance of the postprocessing algorithms.

We propose in this Letter an optimization criterion that results from a compromise between these two antagonistic effects. Taking the classical cubic phase mask as an example [1,2], we will show that such an optimization leads to mask parameters that are significantly different from those reported for the strict invariance of the PSF. The influence of pupil masks on the spectral signal-to-noise ratio (SNR) has been extensively investigated and shows a fundamental limit to the depth-of-field extension $[7,8]$. However, to the best of our knowledge, it is the first time that the
SNR after the deconvolution step is used as a pupil mask optimization criterion.

Let us denote by $O(r)$ the perfect incoherent object of the scene, where $r$ denotes the spatial position, and by $I(r)$ the image intensity obtained with the optical system

$$
I(r)=h_{\psi}(r) * O(r)+n(r),
$$

where the symbol $*$ refers to the convolution operation, $n(r)$ is the measurement noise, and $h_{\psi}(r)$ is the $\mathrm{PSF}$ of the optical system for a given defocus $\psi$ given by

$$
h_{\psi}(r)=\left|F\left\{P(r) \exp \left[i\left(\varphi(r)+\psi r^{2}\right)\right]\right\}\right|^{2},
$$

with

$$
\psi=\frac{\pi R^{2}}{\lambda}\left(\frac{1}{f}-\frac{1}{d_{o}}-\frac{1}{d_{i}}\right),
$$

where $P(r)$ is the pupil function, equal to 1 for $r \leq 1$ and 0 otherwise; $\varphi(r)$ is the phase mask function; $F$ denotes the Fourier transform; $R$ is the radius of the aperture; and $f, d_{o}$, and $d_{i}$ are the focal length, the object distance, and the image sensor plane distance, respectively. The field of view is assumed to be small enough for the optical system to be considered as a linear system. In this Letter, for simplicity's sake, we shall assume that the defocus is constant on the whole scene. However, the method we describe is also valid for $3 \mathrm{D}$ scenes where the distances of objects depend on their positions in the scene.

Our goal is to get the most accurate estimator $\hat{O}(r)=d(r) * I(r)$ of $O(r)$ assuming a linear deconvolution filter $d(r)$. We assume that $n(r)$ and $O(r)$ are stationary random processes, and we denote by $S_{n n}(\nu)$ and $S_{O O}(\nu)$ their respective power spectral densities (PSDs) defined by

$$
S_{n n}(\nu)=|\widetilde{n}(\nu)|^{2}, \quad S_{O O}(\nu)=|O(\nu)|^{2},
$$

where the symbol $\sim$ refers to the Fourier transform and $\nu$ denotes the spatial frequency. 
The amount of noise in the scene is quantified by the input $\mathrm{SNR}_{\text {in }}$ defined as

$$
\left.\left.\mathrm{SNR}_{i n}(\mathrm{~dB})=10 \log _{10}\right\rfloor \int S_{O O}(\nu) \mathrm{d} \nu / \int S_{n n}(\nu) \mathrm{d} \nu\right\rfloor .
$$

The mean squared error (MSE) between the estimate and the true image is

$$
\mathrm{MSE}_{\psi}=\left\langle|\hat{O}(r)-O(r)|^{2}\right\rangle .
$$

This value depends on the defocus $\psi$ since $I(r)$ does. In the definition of the MSE, one has to take care of the translation of the PSF as the defocus $\psi$ varies [1], because the linear term in the phase transfer function varies. In the considered applications, a translation does not affect the image quality and consequently must have no effect on the MSE in Eq. (5). For that purpose, to compute the MSE, we considered a translated version of the PSF defined in Eq. (2), which we denoted $h_{\psi}^{c}$. For any value of $\psi, h_{\psi}^{c}$ is centered at the same location as for $\psi=0$. The translation between $h_{0}$ and $h_{\psi}$ is estimated as the position of the maximum of their cross correlation function. The MSE expression is a function of the signal and noise PSD,

$$
\begin{aligned}
\operatorname{MSE}_{\psi}= & \int\left|\tilde{d}(\nu) \widetilde{h}_{\psi}^{c}(\nu)-1\right|^{2} S_{O O}(\nu) \mathrm{d} \nu \\
& +\int|\tilde{d}(\nu)|^{2} S_{n n}(\nu) \mathrm{d} \nu .
\end{aligned}
$$

It is a sum of two terms corresponding to the inadequate deconvolution filter with the actual PSF and the error caused by the noise.

The criterion we propose to optimize is the MSE averaged over $n_{\text {MSE }}$ defocus values uniformly distributed in the range $\psi_{i} \in\left[0, \psi_{\text {defoc max }}\right]$,

$$
\mathrm{MSE}_{\text {mean }}=\frac{1}{n_{\mathrm{MSE}}} \sum_{i=1}^{n_{\mathrm{MSE}}} \mathrm{MSE}_{\psi_{i}},
$$

and depends on the expression of the deconvolution filter. The deconvolution filter $\widetilde{d}(\nu)$ that minimizes $\mathrm{MSE}_{\text {mean }}$ is calculated by taking the derivative of $\mathrm{MSE}_{\text {mean }}$ with respect to $\widetilde{d}(\nu)$. The solution of this calculus is

$$
\tilde{d}(\nu)=\frac{\frac{1}{n_{d}} \sum_{i=1}^{n_{d}} \widetilde{h}_{\psi_{i}}^{c^{*}}(\nu)}{\frac{1}{n_{d} \sum_{i=1}^{n_{d}}\left|\widetilde{h}_{\psi_{i}}^{c}(\nu)\right|^{2}+S_{n n}(\nu) / S_{O O}(\nu)}} .
$$

We have checked that this optimal filter yields much lower values of $\mathrm{MSE}_{\text {mean }}$ than a Wiener filter that would be designed using only the PSF at focus. This averaged Wiener filter depends on the object and the noise PSD and on the implemented phase mask. In general, neither the object nor the noise PSD is known, so that the filter in Eq. (8) cannot be rigor- ously implemented. However, since our purpose is to study the properties of the pupil mask and not the deconvolution method, we will use the ideal filter in Eq. (8) in the following. We have checked that, if a generic PSD model is used instead of the true one, the results are similar. The filter in Eq. (8) will be computed from $n_{d}$ values of the defocus evenly distributed along the defocus range $\left[0, \psi_{\text {defoc max }}\right]$.

Once the deconvolution filter has been chosen, the MSE depends only on the phase mask. To quantify the imaging system performance, the output SNR $\left(\mathrm{SNR}_{\text {out }}\right)$ is defined as

$$
\mathrm{SNR}_{\text {out }}(\mathrm{dB})=10 \log _{10}\left[\frac{\int S_{\text {OO }}(\nu) \mathrm{d} \nu}{\mathrm{MSE}_{\text {mean }}}\right],
$$

and will be maximized, by optimizing the parameters of the phase mask whose expression is

$$
\varphi(x, y)=\alpha x^{3}+\alpha y^{3},
$$

with $x$ and $y$ being the coordinates of the pupil, normalized to 1 , and $\alpha$ being a mask parameter, in a circular pupil. In our simulation, we considered the object to be a spoke target (ST) that contains high spatial frequencies. The input $\mathrm{SNR}$ is set at $\mathrm{SNR}_{\text {in }}$ $=34 \mathrm{~dB}$, and the maximal defocus value is $\psi_{\text {defoc max }}$ $=15.75$. Considering $n_{d}=5$ defocus values to average the deconvolution filter and $n_{\mathrm{MSE}}=11$ defocus values evenly distributed in the defocus range to estimate $\mathrm{MSE}_{\text {mean }}$, the value of $\mathrm{SNR}_{\text {out }}$ as a function of $\alpha$ is displayed in Fig. 1. The corresponding optimal phase mask parameter of $\alpha=15.74$ leads to $\mathrm{SNR}_{\text {out }}$ $=12.15 \mathrm{~dB}$.

It should be noticed that a slightly different criterion calculated, according to Eq. (11), by taking the smallest SNR in the range $\psi \in\left[0, \psi_{\text {defoc max }}\right]$ has been also investigated and leads to very similar results: optimal parameters $\alpha=17$, corresponding to $\mathrm{SNR}_{\min }$ $=11.04 \mathrm{~dB}$,

$$
\operatorname{SNR}_{\min }(\mathrm{dB})=\min _{i}\left[\mathrm{SNR}_{\psi_{i}}(\mathrm{~dB})\right\rfloor,
$$

with

$$
\mathrm{SNR}_{\psi_{i}}(\mathrm{~dB})=10 \log _{10}\left[\frac{\int S_{O O}(\nu) \mathrm{d} \nu}{\mathrm{MSE}_{\psi_{i}}}\right] .
$$

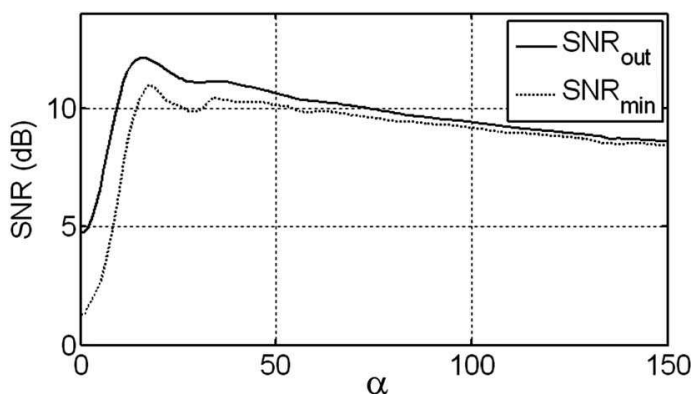

Fig. 1. $\mathrm{SNR}_{\text {out }}$ and $\mathrm{SNR}_{\min }$ as functions of $\alpha$ for ST object with $\mathrm{SNR}_{\text {in }}=34 \mathrm{~dB}$. 

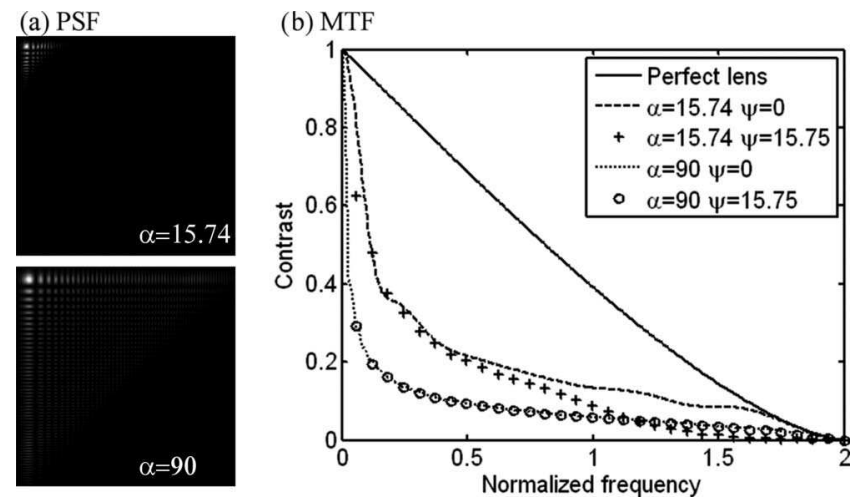

Fig. 2. PSF and MTF at $\alpha=15.74$ and $\alpha=90$.

Figure 2 presents the PSF and the modulation transfer function (MTF) of the imaging system at defocus values $\psi=0$ and $\psi=15.75$ and mask parameter values $\alpha=15.74$ and $\alpha=90$. It can be stated that, at $\alpha$ $=15.74$, the MTF is more sensitive to defocus than at $\alpha=90$ where the response of the optical system is almost strictly invariant. On the other hand, the normalized frequencies between 0 and 1 are less attenuated at $\alpha=15.74$.

Figure 3 gives the deconvoluted images of the ST obtained for both $\alpha$ values. At higher $\alpha$, the results are noisier, as the mask has a lower-pass behavior, increasing the noise after the deconvolution process. On the other hand, the SNR is more constant over the defocus range than at optimal $\alpha$ parameter because the PSF is less sensitive to defocus. The artifact occurring at extremities of the spokes only at lower values of $\alpha$ is related to the filter mismatch be-

(a) Conventional imaging system
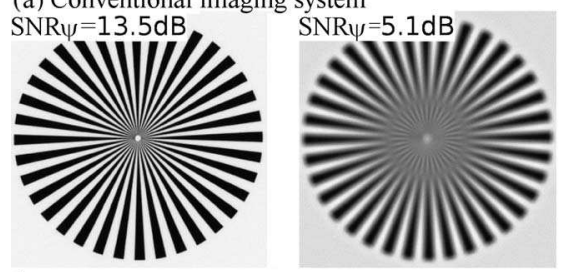

(b) Hybrid imaging system with $\alpha=15.74$
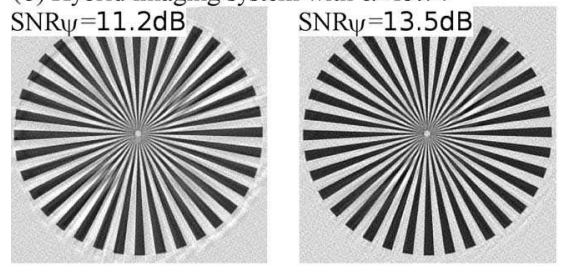

(c) Hybrid imaging system with $\alpha=90$
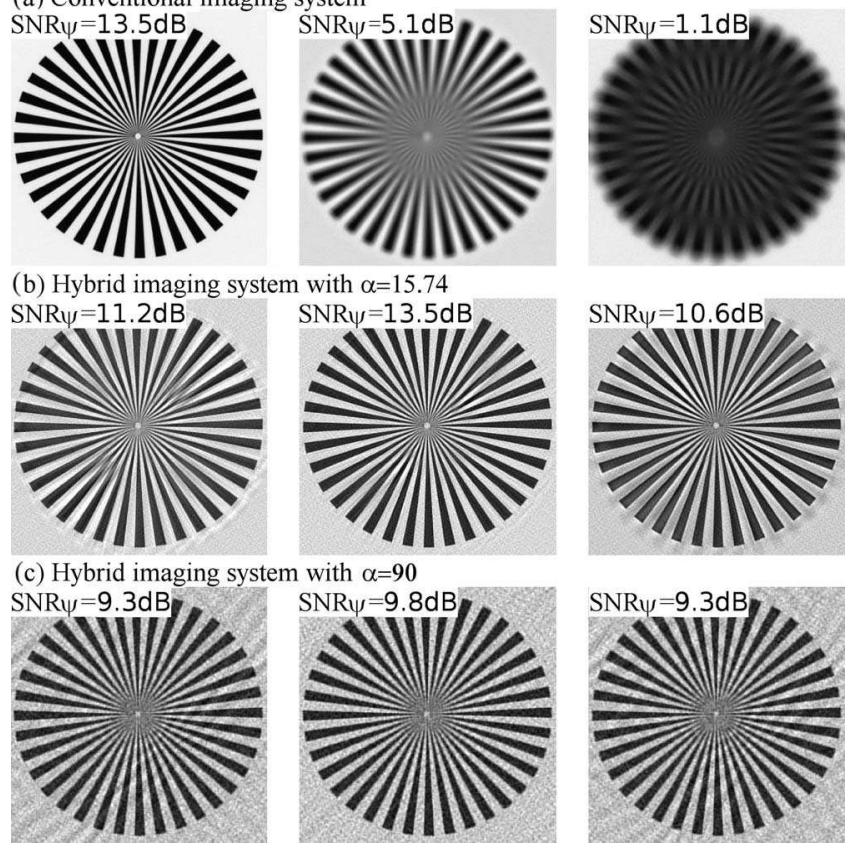

Fig. 3. Results obtained with the ST with (a) a conventional imaging system and with hybrid imaging system at (b) $\alpha=15.74$ and (c) $\alpha=90$. The left column is at $\psi=0$, the middle column is at $\psi=7.62$, and the right column is at $\psi$ $=15.75 . \mathrm{SNR}_{\text {in }}=34 \mathrm{~dB}$.
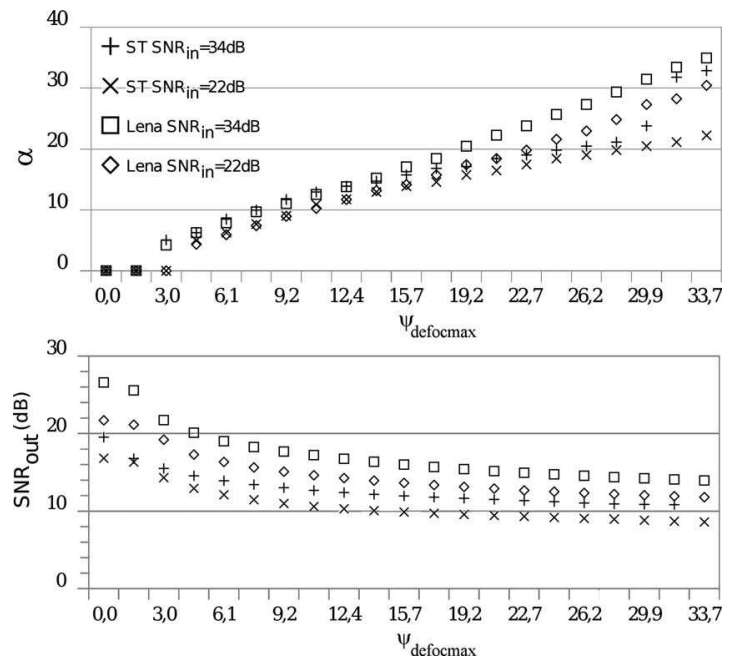

Fig. 4. Optimal parameters versus $\psi_{\text {defoc max }}$ and its corresponding $\mathrm{SNR}_{\text {out }}$, for the ST and Lena.

tween the different PSFs. The optimal value $\alpha$ $=15.74$ is thus a compromise between theses two effects, as the criterion SNR $_{\text {out }}$ has an effective correlation with the visual perception. Consequently, it can be stated that an optimal image quality can be achieved with a nonstrictly invariant PSF.

To illustrate this aspect, we have represented in Fig. 4 the optimal value of $\alpha$ and its corresponding $\mathrm{SNR}_{\text {out }}$ as a function of $\psi_{\text {defoc max }}$ for two different $\mathrm{SNR}_{\text {in }}$ values and two different images: the ST, and the classical "Lena" image, which contains mostly low spatial frequencies. It shows that the optimal value of $\alpha$ increases with $\psi_{\text {defoc max }}$ and with $\mathrm{SNR}_{i n}$.

In conclusion, we defined an optimization criterion that takes into account the noninvariance of the PSF and the noise enhancement induced by the deconvolution process. It results in a trade-off between their two antagonistic effects. As illustrated, this criterion allows one to optimize the parameter of the cubic phase mask and points out that, even if the criterionoptimized phase mask provides a noninvariant PSF, an optimal image quality is achieved. An interesting perspective is to apply the proposed criterion to the comparison of different phase mask designs and to the optimization of hybrid imaging systems.

\section{References}

1. W. T. Cathey and E. R. Dowski, Appl. Opt. 41, 6080 (2002).

2. E. R. Dowski, Jr. and W. T. Cathey, Appl. Opt. 34, 1859 (1995).

3. Q. Yang, L. Liu, and J. Sun, Opt. Commun. 272, 56 (2007).

4. S. S. Sherif, W. T. Cathey, and E. R. Dowski, Appl. Opt. 43, 2709 (2004).

5. N. Caron and Y. Sheng, Appl. Opt. 47, E39 (2008).

6. F. Zhou, G. Li, H. Zhang, and D. Wang, Opt. Lett. 34, 380 (2009).

7. S. S. Sherif, E. R. Dowski, and W. T. Cathey, Opt. Lett. 30, 2566 (2005).

8. S. Bagheri, P. Silveira, and G. Barbastathis, J. Opt. Soc. Am. A 26, 895 (2009). 